\title{
On mass limits for scalar color octet from the LHC data on $t \bar{t}$ invariant mass spectra
}

\author{
M. V. Martynov ${ }^{1, \star}$ and $A$. D. Smirnov ${ }^{1, \star \star}$ \\ ${ }^{1}$ Yaroslavl State University
}

\begin{abstract}
The scalar color octet contribution to the resonance $t \bar{t}$-pair production at the LHC is calculated and analyzed with account of the one loop effective two gluon vertex. From current LHC data on total cross section and invariant mass spectrum of $t \bar{t}$ production we found the $\left(m_{F_{2}}-\sin \beta\right)$-plane exclusion regions.
\end{abstract}

\section{Introduction}

The search for new physics effects beyond the Standard Model (SM) is now one of the goals of the experiments at the LHC. There are many models predicting new physics effects. The most interesting of them look the models predicting new effects due to enlarging the symmetry of the SM because the search for such effects could help us to find the next symmetry in yet unknown hierarchy of the symmetries which possibly unify the known in the SM electroweak and strong interactions of quarks and leptons.

Extended color symmetries are attractive variants of the new physics. One of such variant of physics beyond the SM can be induced by the possible four color symmetry between quarks and leptons of Pati-Salam type [1]. The four color quark-lepton symmetry can be unified with the electroweak $S U_{L}(2) \times U(1)$ symmetry of the SM in the minimal way by the group

$$
G_{M Q L S}=S U_{V}(4) \times S U_{L}(2) \times U_{R}(1)
$$

where the first factor is the vector-like group of the four color quark-lepton symmetry the second one is the usual SM electroweak symmetry group for the left-handed fermions and the third one is the corresponding hypercharge factor for the right-handed fermions (the minimal quark-lepton symmetry model - MQLS-model [2, 3]).

As a result of the Higgs mechanism of splitting the masses of quarks and leptons the four color symmetry in its minimal realization on the gauge group (1) predicts in addition to the SM Higgs doublet $\Phi^{(S M)}$ the existence of the new scalar $S U_{L}(2)$-doublets

$$
\left(\begin{array}{c}
\Phi_{1}^{\prime} \\
\Phi_{2}^{\prime}
\end{array}\right) ;\left(\begin{array}{c}
S_{1 \alpha}^{(+)} \\
S_{2 \alpha}^{(+)}
\end{array}\right) ;\left(\begin{array}{c}
S_{1 \alpha}^{(-)} \\
S_{2 \alpha}^{(-)}
\end{array}\right) ;\left(\begin{array}{c}
F_{1 c} \\
F_{2 c}
\end{array}\right)
$$

^e-mail: martmix@mail.ru

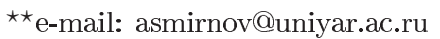


with electric charges

$$
Q_{\Phi}^{e m}: \quad\left(\begin{array}{l}
1 \\
0
\end{array}\right) ;\left(\begin{array}{c}
5 / 3 \\
2 / 3
\end{array}\right) ;\left(\begin{array}{c}
1 / 3 \\
-2 / 3
\end{array}\right) ;\left(\begin{array}{l}
1 \\
0
\end{array}\right)
$$

respectively.

The fields (2) belong to the (1.2.1) +(15.2.1) representation of the group (1), here the fields $\Phi_{1}^{\prime}, \Phi_{2}^{\prime}$ form an additional colorless scalar doublet, $S_{1 \alpha}^{( \pm)}, S_{2 \alpha}^{( \pm)}, \alpha=1,2,3$ are the color triplets forming two scalar leptoquark doublets and the fields $F_{1 c}, F_{2 c}, c=1,2 \ldots 8$ form the scalar doublet of the color octets (the scalar gluon doublet).

Because of their Higgs origin the coupling constants of the doublets (2) with the fermions occur to be proportional to the ratios $m_{f} / \eta$ of the fermion masses $m_{f}$ to the SM VEV $\eta$ and are small for $u$-, $d$-, $s$-quarks are more significant for $c$-, $b$-quarks and are especially significant for $t$-quark $\left(m_{t} / \eta \sim 0.7\right)$. As a result the scalar doublets (2) can manifest themselves more probably in the processes with $t$-quarks. In particular the scalar octet $F_{2}$ could manifest itself as a resonance in $t \bar{t}$-pair production at the LHC. It should be noted that the coupling constants of the doublets (2) with $t$-quark are known (up to the mixing parameters), which gives the possibility to estimate quantitatively the possible effects from these particles in dependence on their masses. The pair production of the scalar octets in $p p$-collisions at the LHC has been discussed in Refs [4-16].

In the present paper we calculate the contribution of the scalar octet to the cross section of the resonance $t \bar{t}$-pair production in pp-collisions and analyse the possibility of manifestation of the scalar gluon $F_{2}$ of the MQLS-model as the corresponding resonance peak in $t \bar{t}$-pair production at the $\mathrm{LHC}$.

\section{MQLS-model scalar color octets - scalar gluons}

The details of interactions of the scalar doublets (2) with quarks and leptons can be found in $[17,18]$. In particular the interaction of the scalar gluon $F_{2}$ with up- and down-quarks in the MQLS-model has the chiral form and can be written as

$$
\begin{aligned}
L_{F_{2} u_{i} u_{j}} & =\bar{u}_{i \alpha}\left[\left(h_{1 F_{2}}^{L}\right)_{i j} P_{L}\right]\left(t_{c}\right)_{\alpha \beta} u_{j \beta} F_{2 c}+\text { h.c. } \\
L_{F_{2} d_{i} d_{j}} & =\bar{d}_{i \alpha}\left[\left(h_{2 F_{2}}^{R}\right)_{i j} P_{R}\right]\left(t_{c}\right)_{\alpha \beta} d_{j \beta} F_{2 c}+\text { h.c. }
\end{aligned}
$$

where $t_{c}, c=1,2 \ldots 8$ are the generators of the $S U_{c}(3)$ group, $P_{L, R}=\left(1 \pm \gamma_{5}\right) / 2$ are the left and right projection operators and $\left(h_{1 F_{2}}^{L}\right)_{i j},\left(h_{2 F_{2}}^{R}\right)_{i j}$ are the Yukawa coupling constants, $i, j=1,2,3$ are the generation indices. As a result of the Higgs mechanism of generating the quark and lepton masses the Yukawa coupling constants $\left(h_{1 F_{2}}^{L}\right)_{i j},\left(h_{2 F_{2}}^{R}\right)_{i j}$ are defined by the expressions

$$
\begin{aligned}
\left(h_{1 F_{2}}^{L}\right)_{i j} & =-\sqrt{3} \frac{1}{\eta \sin \beta}\left[m_{u_{i}}(\delta)_{i j}-\left(K_{1}^{R}\right)_{i k} m_{\nu_{k}}\left(\stackrel{+}{K}_{1}^{L}\right)_{k j}\right], \\
\left(h_{2 F_{2}}^{R}\right)_{i j} & =-\sqrt{3} \frac{1}{\eta \sin \beta}\left[m_{d_{i}}(\delta)_{i j}-\left(K_{2}^{L}\right)_{i k} m_{l_{k}}\left(\stackrel{+}{K}_{2}^{R}\right)_{k j}\right],
\end{aligned}
$$

where $m_{u_{i}}, m_{d_{i}}$ and $m_{\nu_{k}}, m_{l_{k}}$ are the masses of up- and down-quarks and of neutrinos and charged leptons, $K_{1}^{L, R}, K_{2}^{L, R}$ are the mixing matrices in leptoquark currents which are specific for the model with the four color quark-lepton symmetry and $\beta$ is a mixing angle of two 
colorless scalar doublets of MQLS model. Among the coupling constants (5), (6) the largest is the constant $\left(h_{1 F_{2}}^{L}\right)_{33}$ which with neglect of the neutrinos masses takes the form

$$
\left(h_{1 F_{2}}^{L}\right)_{33}=-\sqrt{3} \frac{m_{t}}{\eta \sin \beta} .
$$

The interaction of the scalar gluon $F_{2}$ with $t$-quark can be written as

$$
L_{F_{2} t t}=\bar{t}_{\alpha}\left(h_{F_{2} t \bar{t}}^{S}+h_{F_{2} t \bar{t}}^{P} \gamma_{5}\right)\left(t_{c}\right)_{\alpha \beta} t_{\beta} F_{2 c}+\text { h.c. }
$$

where the correspondent scalar and pseudoscalar coupling constants with account of (7) take the form

$$
h_{F_{2} t \bar{t}}^{S}=h_{F_{2} t \bar{t}}^{P}=-\frac{\sqrt{3}}{2} \frac{m_{t}}{\eta \sin \beta} \approx-0.61 / \sin \beta .
$$

The coupling constants (9) increase with decreasing $\sin \beta$ so that for $\sin \beta=1,0.7,0.4$ the perturbation theory parameters take the values $\left(h_{F_{2} t \bar{t}}^{S, P}\right)^{2} / 4 \pi \approx 0.03,0.06,0.18$ respectively. Below we restrict ourselves by the mixing angle region $0.4 \leq \sin \beta \leq 1$.

The interactions (3), (4) lead to the decays $F_{2} \rightarrow u_{i} \bar{u}_{i}, F_{2} \rightarrow d_{i} \bar{d}_{i}$ and in the case of $m_{F_{2}}>2 m_{t}$ the decay $F_{2} \rightarrow t \bar{t}$ is dominant with the width $[17,18]$

$$
\Gamma\left(F_{2} \rightarrow t \bar{t}\right)=m_{F_{2}} \frac{3}{32 \pi}\left(\frac{m_{t}}{\eta}\right)^{2}\left(1-2 \frac{m_{t}^{2}}{m_{F_{2}}^{2}}\right) \sqrt{1-4 \frac{m_{t}^{2}}{m_{F_{2}}^{2}}} \frac{1}{\sin ^{2} \beta} .
$$

For the masses $m_{F_{2}}=400-2000 \mathrm{GeV}$ the width (10) is of about $(2-30) / \sin ^{2} \beta \mathrm{GeV}$ and $\Gamma_{F_{2}} / m_{F_{2}}=(0.5-1.5) \% / \sin ^{2} \beta$.

As seen from the expressions (5), (6) the coupling constants of the interaction of the scalar gluon $F_{2}$ with $u$ - and $d$ - quarks are of order of $m_{u} / \eta \sim m_{d} / \eta \sim 10^{-5}$ and the interactions of these quarks as the initial partons with the scalar gluon $F_{2}$ are negligibly small. On the other hand the Lagrangian (8), (9) can induce through the loop contribution of $t$-quark the more significant effective interaction of two initial gluons with the scalar gluon $F_{2}$, which should be taken into account. The analogous effective two gluon interaction is induced also with the colorless scalar $\Phi_{2}^{\prime}$.

\section{Resonance contribution of scalar color octet to $t \bar{t}$ production}

The calculation of the effective two gluon vertex of interaction with the scalar octet is like to that with the colorless scalar and we perform below these calculations simultaneously. For this purpose we write the flavour diagonal interactions of scalar octet and of the scalar color singlet with quarks in the model independent form as

$$
L_{\Phi q \bar{q}}=\bar{q}_{\alpha}\left(h_{\Phi q \bar{q}}^{S}+h_{\Phi q \bar{q}}^{P} \gamma_{5}\right) \Phi_{\alpha \beta} q_{\beta}+\text { h.c. },
$$

where $\Phi_{\alpha \beta}=\Phi_{0} \delta_{\alpha \beta}$ for the colorless scalar particle $\Phi_{0}$ and $\Phi_{\alpha \beta}=\Phi_{8 c}\left(t_{c}\right)_{\alpha \beta}$ for the scalar octet $\Phi_{8}, t_{c}$ are the generators of the $S U_{c}(3) \operatorname{group}(c=1,2 \ldots 8), h_{\Phi q \bar{q}}^{S}$ and $h_{\Phi q \bar{q}}^{P}$ are the corresponding scalar and pseudoscalar coupling constants. For the MQLS-model the scalars $\Phi_{8}$ and $\Phi_{0}$ correspond to $F_{2}$ and $\Phi_{2}^{\prime}$ respectively.

The effective vertex $\Gamma_{a b \Phi}^{(q) \mu \nu}\left(p, k_{1}, k_{2}\right)$ of interaction of two gluons with scalar field $\Phi=$ $\Phi_{0}, \Phi_{8 c}$ induced by the Lagrangian (11) with account of one loop contribution of quark $q$ is described by the diagrams in the Fig. 1 


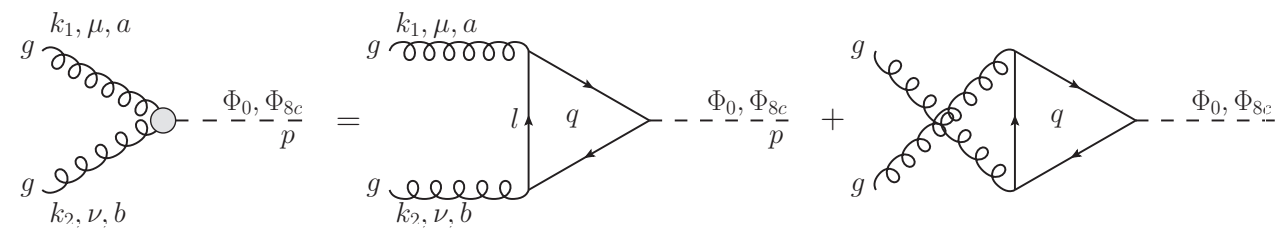

Figure 1. The effective vertex $\Gamma_{a b \Phi}^{(q) \mu \nu}\left(p, k_{1}, k_{2}\right)$ induced by one loop contribution of quark $q$ for $\Phi=\Phi_{0}, \Phi_{8 c}$.

With account of the contributions of all the quarks the resulted effective vertex $\Gamma_{a b \Phi}^{\mu \nu}\left(p, k_{1}, k_{2}\right)$ in the case of real gluons $\left(k_{1}^{2}=0, k_{2}^{2}=0, \hat{s}=p^{2}=2\left(k_{1} k_{2}\right)\right)$ can be parametrized as [19]:

$$
\begin{aligned}
& \Gamma_{a b \Phi}^{\mu \nu}\left(p, k_{1}, k_{2}\right)=\sum_{q} \Gamma_{a b \Phi}^{(q) \mu \nu}\left(p, k_{1}, k_{2}\right)= \\
& =-C_{a b \Phi} \frac{\alpha_{s} \sqrt{\hat{s}}}{\pi}\left[\left(g^{\mu \nu}-\frac{2 k_{1}^{\nu} k_{2}^{\mu}}{\hat{s}}\right) F_{\Phi}^{S}(\hat{s})-2 i \varepsilon^{\mu \nu \rho \sigma} \frac{k_{1 \rho} k_{2 \sigma}}{\hat{s}} F_{\Phi}^{P}(\hat{s})+\frac{2 k_{1}^{\mu} k_{2}^{\nu}}{\hat{s}} G_{\Phi}^{S}(\hat{s})\right]
\end{aligned}
$$

by the form factors

$$
F_{\Phi}^{S, P}(\hat{s})=\sum_{q} h_{\Phi q \bar{q}}^{S, P} \tilde{F}^{S, P}\left(\hat{s}, m_{q}^{2}\right), \quad G_{\Phi}^{S}(\hat{s})=\sum_{q} h_{\Phi q \bar{q}}^{S} \tilde{G}^{S}\left(\hat{s}, m_{q}^{2}\right),
$$

where $C_{a b \Phi}$ is the color factor with

$$
C_{a b \Phi_{0}}=\delta_{a b} / 2 \equiv C_{a b}, \quad C_{a b \Phi_{8 c}}=d_{a b c} / 4 \equiv C_{a b c}
$$

for the color singlet $\Phi_{0}$ and for the color octet $\Phi_{8}$.

For the form factors $\tilde{F}^{S, P}\left(\hat{s}, m_{q}^{2}\right), \tilde{G}^{S}\left(\hat{s}, m_{q}^{2}\right)$ we have found the expressions

$$
\begin{aligned}
& \tilde{F}^{S}\left(\hat{s}, m_{q}^{2}\right)=\frac{m_{q}}{\sqrt{\hat{s}}}\left[\left(\hat{s}-4 m_{q}^{2}\right) C_{0}\left(0,0, \hat{s}, m_{q}^{2}, m_{q}^{2}, m_{q}^{2}\right)-2\right] \equiv \tilde{F}^{S}\left(\rho_{q}\right), \\
& \tilde{F}^{P}\left(\hat{s}, m_{q}^{2}\right)=m_{q} \sqrt{\hat{s}} C_{0}\left(0,0, \hat{s}, m_{q}^{2}, m_{q}^{2}, m_{q}^{2}\right) \equiv \tilde{F}^{P}\left(\rho_{q}\right), \\
& \tilde{G}^{S}\left(\hat{s}, m_{q}^{2}\right)= \\
& =\frac{m_{q}}{\sqrt{\hat{s}}}\left[\left(\hat{s}+4 m_{q}^{2}\right) C_{0}\left(0,0, \hat{s}, m_{q}^{2}, m_{q}^{2}, m_{q}^{2}\right)+4 B_{0}\left(\hat{s}, m_{q}^{2}, m_{q}^{2}\right)-\frac{4 A_{0}\left(m_{q}^{2}\right)}{m_{q}^{2}}\right] \equiv \tilde{G}^{S}\left(\rho_{q}\right),
\end{aligned}
$$

where $A_{0}, B_{0}, C_{0}$ are the Passarino-Veltman (PV) scalar integrals [20, 21], with account of the explicit form of these integrals the form factors (15)-(17) depend only on the variable $\rho_{q}=$ $\sqrt{\hat{s}} / m_{q}$. The form factors (15)-(17) account the one loop contribution of quark $q$ in the model independent way, the specific features of the model are presented by the coupling constants $h_{\Phi q \bar{q}}^{S, P}$ in the form factors (13). Due to the gauge invariance the longitudinal component of the vertex (12) parametrized by the form factor $G_{\Phi}^{S}(\hat{s})$ do not enter to the observed variables.

We have calculated the cross section of the process $g g \rightarrow Q \bar{Q}$ of $Q \bar{Q}$ pair production in gluon fusion in QCD LO with account also of the effective vertex (12) in Ref. [19]. The diagrams of this process are shown in the Fig. 2. 

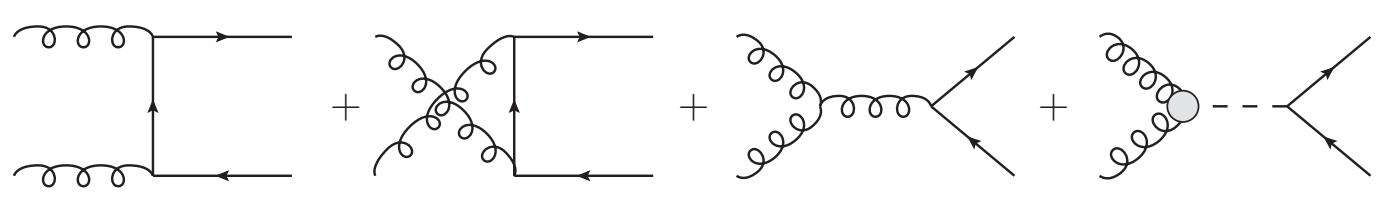

Figure 2. Diagrams of the process $g g \rightarrow Q \bar{Q}$ in the SM LO and with account of the effective vertex $\Gamma_{a b \Phi}^{\mu \nu}\left(p, k_{1}, k_{2}\right)$.

The total cross section of the process $g g \rightarrow Q \bar{Q}$ can be written as the sum

$$
\sigma_{0}(g g \rightarrow Q \bar{Q}, \mu)=\sigma_{0}^{\mathrm{SM}}(g g \rightarrow Q \bar{Q}, \mu)+\Delta \sigma^{\Phi}(g g \rightarrow Q \bar{Q}, \mu)
$$

of the well known QCD LO cross section

$$
\sigma_{0}^{\mathrm{SM}}(g g \rightarrow Q \bar{Q}, \mu)=\frac{\alpha_{s}^{2}(\mu) \pi}{48 \hat{s}}\left[\left(v^{4}-18 v^{2}+33\right) \log \frac{1+v}{1-v}+v\left(31 v^{2}-59\right)\right],
$$

where $v=\sqrt{1-4 m_{Q}^{2} / \hat{s}}$ is the velocity of quark $Q$ in the center of mass frame, $\hat{s}$ is the squared energy in the center of momentum frame of the gluons, $\mu$ is a typical mass scale of the process, and the contribution $\Delta \sigma^{\Phi}(g g \rightarrow Q \bar{Q}, \mu)$ to this process from the scalar $\Phi$.

For the contribution $\Delta \sigma^{\Phi}(g g \rightarrow Q \bar{Q}, \mu)$ we have found the expression

$$
\begin{aligned}
& \Delta \sigma^{\Phi}(g g \rightarrow Q \bar{Q}, \mu)= \\
& =\frac{\tilde{C}_{\Phi}^{(1)}}{64} \frac{\alpha_{s}^{2}(\mu) m_{Q}}{\pi \sqrt{\hat{s}}} \frac{\operatorname{Re}\left[( \hat { s } - m _ { \Phi } ^ { 2 } - i m _ { \Phi } \Gamma _ { \Phi } ) \left(-h_{\left.\left.\Phi Q \bar{Q}^{S^{*}} v^{2} F_{\Phi}^{S}(\hat{s})-h_{\Phi Q \bar{Q}}^{P^{*}} F_{\Phi}^{P}(\hat{s})\right)\right]}^{\left(\hat{s}-m_{\Phi}^{2}\right)^{2}+m_{\Phi}^{2} \Gamma_{\Phi}^{2}} \log \frac{1+v}{1-v}+\right.\right.}{+} \\
& +\frac{\tilde{C}_{\Phi}^{(2)}}{1024} \frac{\alpha_{s}^{2}(\mu) v \hat{s}}{\pi^{3}} \frac{\left|h_{\Phi Q \bar{Q}}^{S}\right|^{2} v^{2}+\left|h_{\Phi Q \bar{Q}}^{P}\right|^{2}}{\left(\hat{s}-m_{\Phi}^{2}\right)^{2}+m_{\Phi}^{2} \Gamma_{\Phi}^{2}}\left(\left|F_{\Phi}^{S}(\hat{s})\right|^{2}+\left|F_{\Phi}^{P}(\hat{s})\right|^{2}\right)
\end{aligned}
$$

where the form factors $F_{\Phi}^{S, P}(\hat{s})$ are given by the expressions $(13),(15),(16)$ and $\tilde{C}_{\Phi}^{(1)}, \tilde{C}_{\Phi}^{(2)}$ are the color factors with

$$
\begin{array}{ll}
\tilde{C}_{\Phi_{0}}^{(1)}=C_{a b} C_{a b}=2, & \tilde{C}_{\Phi_{0}}^{(2)}=C_{a b} C_{a b} n_{c}=6, \\
\tilde{C}_{\Phi_{8}}^{(1)}=C_{a b c} C_{a b c}=5 / 6, & \tilde{C}_{\Phi_{8}}^{(2)}=C_{a b c} C_{a b c} / 2=5 / 12
\end{array}
$$

for the color singlet $\Phi_{0}$ and for the color octet $\Phi_{8}$ respectively, $n_{c}$ is the number of colors of the $S U_{c}\left(n_{c}\right)$ group, the numerical values in (22), (23) correspond to the $S U_{c}(3)$ group.

The total cross section $\sigma_{t o t}(p p \rightarrow t \bar{t})$ of the $t \bar{t}$ production in $p p$-collisions with account of the contribution of scalar octet $F_{2}$ can be written as the sum

$$
\sigma_{t o t}(p p \rightarrow t \bar{t})=\sigma^{\mathrm{SM}}(p p \rightarrow t \bar{t})+\Delta \sigma^{F_{2}}(p p \rightarrow t \bar{t})
$$

of the SM cross section $\sigma^{\mathrm{SM}}(p p \rightarrow t \bar{t})$ and the contribution $\Delta \sigma^{F_{2}}(p p \rightarrow t \bar{t})$ induced by scalar gluon $F_{2}$ via effective vertex (12)-(16). 
Table 1. Summary of the most precise ATLAS and CMS measurements of the total $t \bar{t}$ cross sections at 7,8 and $13 \mathrm{TeV}$.

\begin{tabular}{|c|c|c|c|c|}
\hline Exp. & $\overline{\sqrt{s}[\mathrm{TeV}]}$ & $\mathcal{L}\left[\mathrm{fb}^{-1}\right]$ & $\sigma^{\text {tot }}(t \bar{t})[\mathrm{pb}]$ & Ref. \\
\hline \multirow{3}{*}{ ATLAS } & 7 & 4.6 & $182.9 \pm 3.1(\mathrm{stat}) \pm 4.2(\mathrm{sys}) \pm 3.6(\mathrm{lumi}) \pm 3.3(\mathrm{bm})$ & {$[23]$} \\
\hline & 8 & 20.3 & $242.4 \pm 1.7($ stat $) \pm 5.5(\mathrm{sys}) \pm 7.5(\mathrm{lumi}) \pm 4.2(\mathrm{bm})$ & {$[23]$} \\
\hline & 13 & 3.2 & $818 \pm 8$ (stat) \pm 27 (sys) \pm 19 (lumi) $\pm 12(\mathrm{bm})$ & [24] \\
\hline \multirow{3}{*}{ CMS } & 7 & 5.0 & $173.6 \pm 2.1(\text { stat })_{-4.0}^{+4.5}(\mathrm{sys}) \pm 3.8$ (lumi) & {$[25]$} \\
\hline & 8 & 19.7 & $244.9 \pm 1.4(\text { stat })_{-5.5}^{+6.3}(\mathrm{sys}) \pm 6.4($ lumi $)$ & {$[25]$} \\
\hline & 13 & 2.3 & $835 \pm 3($ stat $) \pm 23($ sys $) \pm 23$ (lumi) & {$[26]$} \\
\hline
\end{tabular}

We obtain the total cross section from partonic cross sections (19), (21) by integrating the expression

$$
\begin{aligned}
& \frac{d \sigma_{t o t}(p p \rightarrow t \bar{t})}{d x_{1} d x_{2}}=\sum_{k} F_{k}^{p \bar{p}}\left(x_{1}, x_{2}, \mu_{f}\right) K(s) \sigma_{0}^{S M}\left(\overline{q_{k}} q_{k} \rightarrow t \bar{t}, \mu\right)+ \\
& +F_{g}^{p p}\left(x_{1}, x_{2}, \mu_{f}\right) K(s) \sigma_{0}^{S M}(g g \rightarrow t \bar{t}, \mu)+F_{g}^{p p}\left(x_{1}, x_{2}, \mu_{f}\right) \Delta \sigma^{F_{2}}(g g \rightarrow t \bar{t}, \mu)
\end{aligned}
$$

over the variables $0 \leq x_{1}, x_{2} \leq 1$, where $x_{1}, x_{2}$ are partonic parts of the momenta of protons, $\hat{s}=x_{1} x_{2} s, s=\left(P_{1}+P_{2}\right)^{2}, P_{1}, P_{2}$ are the momenta of the colliding protons, $\sigma_{0}^{S M}\left(\overline{q_{k}} q_{k} \rightarrow t \bar{t}, \mu\right)$ is the well known SM LO cross section, $K(s)$ is the $K$-factor, which we use for the better agreement of the SM LO predictions of the cross section of $t \bar{t}$-pair production with the corresponding aNNNLO SM predictions [22]. The partonic functions in (25) are given as

$$
\begin{aligned}
& F_{k}^{p p}\left(x_{1}, x_{2}, \mu_{f}\right)=f_{q_{k}}^{p}\left(x_{1}, \mu_{f}\right) f_{\bar{q}_{k}}^{p}\left(x_{2}, \mu_{f}\right)+f_{\bar{q}_{k}}^{p}\left(x_{1}, \mu_{f}\right) f_{q_{k}}^{p}\left(x_{2}, \mu_{f}\right), \\
& F_{g}^{p p}\left(x_{1}, x_{2}, \mu_{f}\right)=f_{g}^{p}\left(x_{1}, \mu_{f}\right) f_{g}^{p}\left(x_{2}, \mu_{f}\right),
\end{aligned}
$$

where $f_{q_{k}}^{p}\left(x, \mu_{f}\right), f_{\bar{q}_{k}}^{p}\left(x, \mu_{f}\right), f_{g}^{p}\left(x, \mu_{f}\right)$ are the parton distribution functions of quark $q_{k}$ of flavor $k$, antiquark $\bar{q}_{k}$ and gluons in the proton, $\mu_{f}$ is the factorization scale.

For numerical calculations we use the analytical expressions for scalar Passarino-Veltman integrals $A_{0}, B_{0}, C_{0}$ from the Ref. [21] and we also perform the cross check with using numerical package LoopTools/FF [27, 28].

For calculations we use the parton distribution functions MMHT 2014 [29] (NNLO, $\mu=$ $\left.\mu_{f}=m_{t}, m_{t}=173.21 \mathrm{GeV}\right)$, with the values of $K$-factors $K(s)=1.6687,1.6752,1.6833$ for energies $\sqrt{s}=7,8,13 \mathrm{TeV}$ respectively, in this case the cross section $\sigma^{\mathrm{SM}}(p p \rightarrow t \bar{t})$ reproduces well the aNNNLO SM predictions for the cross section of $t \bar{t}$ production [22] Also we perform cross check our partons integrations with use PDFs CT14 and METAv10 in the ManeParse. (package for the Wolfram Mathematica for parsing various PDF functions) [30], the difference between these results was about $1 \%$.

We have calculated the contributions $\Delta \sigma^{F_{2}}(p p \rightarrow t \bar{t})$ to the total cross section of the $t \bar{t}$ production from the scalar gluon $F_{2}$ defined by the last term in the expression (25) for $\sqrt{s}=7,8,13 \mathrm{TeV}, m_{F_{2}}=400 \div 1000 \mathrm{GeV}$ and $\sin \beta=0.4 \div 1, \Delta \sigma^{F_{2}}(p p \rightarrow t \bar{t})$ varies from 0.3 to $54.9 \mathrm{pb}$ (depending on $\sqrt{s}, m_{F_{2}}, \sin \beta$ ) [19].

From current most precise ATLAS and CMS measurements of the total $t \bar{t}$ cross sections at 7,8 and $13 \mathrm{TeV}$ (Table 1$)$ we found exclusion area at the $\left(m_{F_{2}}-\sin \beta\right)$-plane, the area is shown in the Fig. 3. 


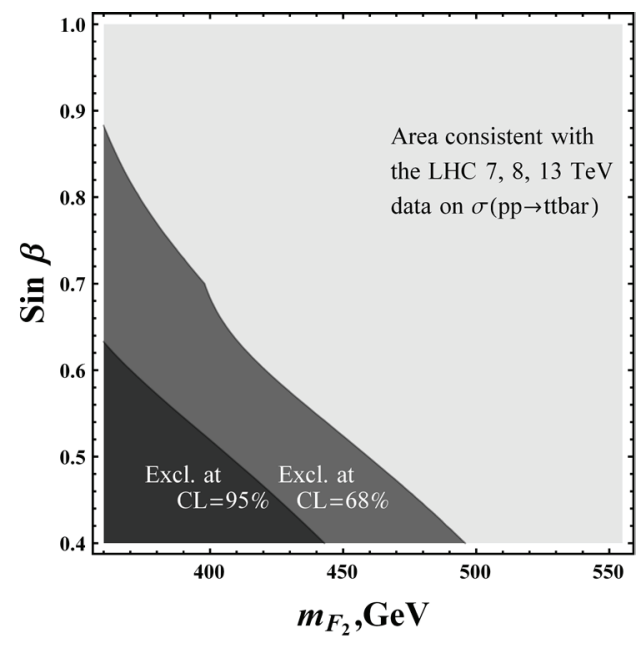

Figure 3. The $\left(m_{F_{2}}-\sin \beta\right)$-plane exclusion regions (at $95 \%$ and $68 \%$ probability level) resulting from the LHC data on total $t \bar{t}$ cross sections at 7, 8 and $13 \mathrm{TeV}[23-26]$.

Using the known relations between the variables $x_{1}, x_{2}$ and the invariant mass $m_{t \bar{t}}$ of $t \bar{t}$-pair and the rapidity $y$ of the final $t$-quark

$$
m_{t \bar{t}}^{2}=x_{1} x_{2} s, \quad y=\ln \frac{x_{1}}{x_{2}}, \quad x_{1,2}=\frac{m_{t \bar{t}}}{\sqrt{s}} e^{ \pm y / 2}
$$

and integrating the expression (25) over the rapidity $y$ with account of the scalar gluon contribution $\Delta \sigma^{F_{2}}\left(g g \rightarrow t \bar{t}, \mu_{f}\right)$ we obtain the invariant mass spectrum $d \sigma(p p \rightarrow t \bar{t}) / d m_{t \bar{t}}$ in the form

$$
\frac{d \sigma_{t o t}(p p \rightarrow t \bar{t})}{d m_{t \bar{t}}}=\frac{m_{t \bar{t}}}{s} \int_{-\ln \left(s / m_{t \bar{t}}^{2}\right)}^{+\ln \left(s / m_{t \bar{t}}^{2}\right)} \frac{d \sigma_{t o t}(p p \rightarrow t \bar{t})}{d x_{1} d x_{2}} d y
$$

In the same way but with neglect of the scalar gluon contribution $\Delta \sigma^{F_{2}}\left(g g \rightarrow t \bar{t}, \mu_{f}\right)$ we obtain the background invariant mass spectrum $d \sigma_{b}(p p \rightarrow t \bar{t}) / d m_{t \bar{t}}$ which is in agreement with the theoretical predictions [31] and with the experimental results [32-34].

With using experimental data on the $t \bar{t}$ invariant mass spectrum (LHC ATLAS $8 \mathrm{TeV}$, $\left.\mathrm{L}=20.3 \mathrm{fb}^{-1}[35]\right)$ we found exclusion area at the $\left(m_{F_{2}}-\sin \beta\right)$-plane, that is shown in the Fig. 4.

In conclusion, we summarize the results of this paper.

The effective vertex of interaction of the scalar color octet with two gluons is calculated with account of the one loop quark contribution. With account of this interaction the contribution of the scalar color octet to the partonic cross section of resonance $Q \bar{Q}$-pair production in the gluon fusion is calculated.

The total and differential cross sections of the $t \bar{t}$ production in $p p$-collisions at the LHC are calculated with account of the resonance contribution of scalar color octet $F_{2}$ predicted by the minimal model with the four color quark-lepton symmetry and analyzed in dependence on two parameters of the model, the $F_{2}$ mass $m_{F_{2}}$ and mixing angle $\beta$. 


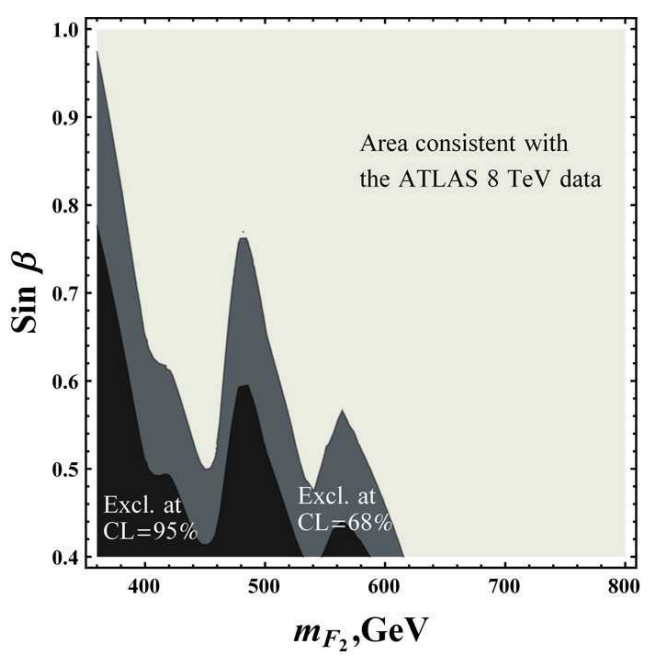

Figure 4. The $\left(m_{F_{2}}-\sin \beta\right)$-plane exclusion regions (at $95 \%$ and $68 \%$ probability level) resulting from the LHC ATLAS data on $t \bar{t}$ invariant mass spectrum at $8 \mathrm{TeV}$ [35].

From the comparison with the LHC data on total (at $\sqrt{s}=7,8,13 \mathrm{TeV}$ ) and the differential cross sections (at $\sqrt{s}=8 \mathrm{TeV}$ ) of $t \bar{t}$ production it is shown that there is region of $\left(m_{F_{2}}-\sin \beta\right.$ )plane of exclusion by these data. But for $\sin \beta=1$ and for all the masses $m_{F_{2}}$ the scalar color octet $F_{2}$ gives the contribution to this process of about a few percents and can not be visible in these data.

The work is done within "YSU Initiative Scientific Researches" (Project No. AAAA-A16116070610023-3)

\section{References}

[1] J.C. Pati, A. Salam, Phys. Rev. D 10, 275 (1974)

[2] A.D. Smirnov, Phys. Lett. B 346, 297 (1995), hep-ph/9503239

[3] A.D. Smirnov, Phys. At. Nucl. 58, 2137 (1995), [Yad. Fiz. 58, 2252 (1995)]

[4] A.V. Manohar, M.B. Wise, Phys. Rev. D74, 035009 (2006), hep-ph/0606172

[5] M.I. Gresham, M.B. Wise, Phys. Rev. D76, 075003 (2007), 0706.0909

[6] M. Gerbush, T.J. Khoo, D. Phalen, A. Pierce, D. Tucker-Smith, Phys. Rev. D77, 095003 (2008), 0710.3133

[7] A.R. Zerwekh, C.O. Dib, R. Rosenfeld, Phys. Rev. D77, 097703 (2008), 0802.4303

[8] P.F. Perez, H. Iminniyaz, G. Rodrigo, Phys. Rev. D78, 015013 (2008), 0803.4156

[9] S.Y. Choi, M. Drees, J. Kalinowski, J.M. Kim, E. Popenda, P.M. Zerwas, Phys. Lett. B672, 246 (2009), 0812.3586

[10] M.V. Martynov, A.D. Smirnov, Mod. Phys. Lett. A23, 2907 (2008), 0807.4486

[11] M.V. Martynov, A.D. Smirnov, Phys. At. Nucl. 73, 1207 (2010)

[12] D. Goncalves-Netto, D. Lopez-Val, K. Mawatari, T. Plehn, I. Wigmore, Phys. Rev. D85, 114024 (2012), 1203.6358 
[13] A. Idilbi, C. Kim, T. Mehen, Phys. Rev. D82, 075017 (2010), 1007.0865

[14] S. Calvet, B. Fuks, P. Gris, L. Valery, JHEP 04, 043 (2013), 1212.3360

[15] J. Kubo, K.S. Lim, M. Lindner, Phys. Rev.Lett. 113, 091604 (2014), 1403.4262

[16] G. Grilli di Cortona, E. Hardy, A.J. Powell, JHEP 08, 014 (2016), 1606.07090

[17] P.Y. Popov, A.V. Povarov, A.D. Smirnov, Mod. Phys. Lett. A20, 3003 (2005), hep-ph/0511149

[18] A.V. Povarov, P.Yu. Popov, A.D. Smirnov, Phys. Atom. Nucl. 70, 739 (2007), [Yad. Fiz.70,771(2007)]

[19] I.V. Frolov, M.V. Martynov, A.D. Smirnov, Mod. Phys. Lett. A31, 1650224 (2016), 1610.08409

[20] G. Passarino, M.J.G. Veltman, Nucl. Phys. B160, 151 (1979)

[21] A. Denner, Fortschr. Phys. 41, 307 (1993), 0709.1075

[22] N. Kidonakis, Phys. Rev. D90, 014006 (2014), 1405.7046

[23] G. Aad et al. (ATLAS), Eur. Phys. J. C74, 3109 (2014), [Addendum: Eur. Phys. J.C76,no.11,642(2016)], 1406.5375

[24] M. Aaboud et al. (ATLAS), Phys. Lett. B761, 136 (2016), 1606.02699

[25] V. Khachatryan et al. (CMS), JHEP 08, 029 (2016), 1603.02303

[26] A.M. Sirunyan et al. (CMS) (2017), 1701.06228

[27] T. Hahn, M. Perez-Victoria, Comput. Phys. Commun. 118, 153 (1999), hep-ph/9807565

[28] G.J. van Oldenborgh, Comput. Phys. Commun. 66, 1 (1991)

[29] L. Harland-Lang, A. Martin, P. Motylinski, R. Thorne, Eur.Phys.J. C75, 204 (2015), 1412.3989

[30] D.B. Clark, E. Godat, F.I. Olness, Comput. Phys. Commun. 216, 126 (2017), 1605.08012

[31] V. Ahrens, A. Ferroglia, M. Neubert, B.D. Pecjak, L.L. Yang, JHEP 1009, 097 (2010), 1003.5827

[32] G. Aad et al. (ATLAS), Phys. Rev. D90, 072004 (2014), 1407.0371

[33] S. Chatrchyan et al. (CMS), Eur.Phys.J. C73, 2339 (2013), 1211.2220

[34] V. Khachatryan et al. (CMS), Eur. Phys. J. C75, 542 (2015), 1505.04480

[35] G. Aad et al. (ATLAS), Eur. Phys. J. C76, 538 (2016), 1511.04716 